\title{
ENUMERATION OF SET-THEORETIC SOLUTIONS TO THE YANG-BAXTER EQUATION
}

\author{
Ö. AKGÜN, M. MEREB, L. VENDRAMIN
}

\begin{abstract}
We use Constraint Satisfaction methods to enumerate and construct set-theoretic solutions to the Yang-Baxter equation of small size. We show that there are 321,931 involutive solutions of size nine, 4,895,272 involutive solutions of size ten and 422,449,480 non-involutive solution of size eight. Our method is then used to enumerate noninvolutive biquandles.
\end{abstract}

\section{INTRODUCTION}

The Yang-Baxter equation (YBE) was first introduced in the field of statistical mechanics and for several decades it has been studied in mathematics and physics $[5,41]$. Recent progress in set-theoretic solutions to the YBE shed new light on the importance of this equation in algebra and combinatorics [13, 18, 19, 21, 25, 27, 34, 38].

A set-theoretic solution to the YBE is a pair $(X, r)$, where $X$ is a set and $r: X \times X \rightarrow X \times X$ is a bijective map such that

$$
(r \times \text { id })(\text { id } \times r)(r \times \text { id })=(\text { id } \times r)(r \times \text { id })(\text { id } \times r)
$$

holds, where the juxtaposition denotes the usual composition of maps. Note that this is a functional equation in the space of maps $X^{3} \rightarrow X^{3}$, where $X^{3}=X \times X \times X$.

Example 1.1 (permutation solutions over sets). If $X$ is a non-empty set and $\sigma: X \rightarrow X$ and $\tau: X \rightarrow X$ are bijections, then the pair $(X, r)$, where

$$
r: X \times X \rightarrow X \times X, \quad r(x, y)=(\sigma(y), \tau(x)),
$$

is a set-theoretical solution if and only if $\sigma$ and $\tau$ commute. Indeed, on the one hand,

$$
\begin{aligned}
(r \times \mathrm{id})(\mathrm{id} \times r)(r \times \mathrm{id})(x, y, z) & =(r \times \mathrm{id})(\mathrm{id} \times r)(\sigma(y), \tau(x), z) \\
& =(r \times \mathrm{id})\left(\sigma(y), \sigma(z), \tau^{2}(x)\right) \\
& =\left(\sigma^{2}(z), \tau \sigma(y), \tau^{2}(x)\right)
\end{aligned}
$$

and, on the other hand,

$$
\begin{aligned}
(\mathrm{id} \times r)(r \times \mathrm{id})(\mathrm{id} \times r)(x, y, z) & =(\mathrm{id} \times r)(r \times \mathrm{id})(x, \sigma(z), \tau(y)) \\
& =(\mathrm{id} \times r)\left(\sigma^{2}(z), \tau(x), \tau(y)\right) \\
& =\left(\sigma^{2}(z), \sigma \tau(y), \tau^{2}(x)\right) .
\end{aligned}
$$

2010 Mathematics Subject Classification. Primary:16T25; Secondary: 81R50. Key words and phrases. Yang-Baxter, biquandles, constraint programming. 
We say that the solutions $(X, r)$ and $(Y, s)$ are isomorphic if there is a bijective map $f: X \rightarrow Y$ such that

$$
(f \times f) r=s(f \times f) .
$$

From the combinatorial perspective certain types of solutions are particularly important; these are called non-degenerate solutions. By convention, if $(X, r)$ is a set-theoretic solution to the YBE, we write

$$
r(x, y)=\left(\sigma_{x}(y), \tau_{y}(x)\right) .
$$

The solution $(X, r)$ is then said to be non-degenerate if the maps $\sigma_{x}$ and $\tau_{x}$ are bijective for all $x \in X$.

Convention 1.2. A solution will always be a non-degenerate set-theoretic solution to the YBE. We will only consider finite solutions.

Set-theoretic solutions to the YBE attracted a lot of attention and lead to several interesting connections between group theory, ring theory and combinatorics. The combinatorial version of the celebrated Yang-Baxter equation was first formulated by Drinfel'd in [12] and considered later in [13,21] for involutive solutions and in $[27,37]$ for arbitrary solutions. Set-theoretic solutions are known to have deep connections with bijective 1-cocycles, ordered groups, groups of I-type, regular subgroups, radical rings, skew braces, nil rings, homology theory, Hopf-Galois extensions [9, 10,34,36].

The main result in this article is an explicit classification of solutions to the YBE of small size. This is achieved by using some combinatorial ideas closely connected to the Yang-Baxter equation, Constraint Satisfaction methods $[15,22]$ and, in particular, the constraint modelling assistant Savile Row [31], and the computational algebra package GAP [16]. Similar techniques have been used to enumerate semi-groups of order $\leqslant 10$, see [11].

The combination of these techniques allows us to build a huge database of involutive and non-involutive solutions to the YBE, a good and useful source of examples that gives an explicit and direct way to approach some open problems concerning the YBE. The database is freely available as a library for GAP at https://github.com/vendramin/enumeration, with DOI name 10.5281/zenodo. 5180745 .

In [13] Etingof, Schedler and Soloviev constructed all involutive solutions of size $\leqslant 8$. We summarize our findings on involutive solutions in the following statement.

\section{Theorem 1.3.}

(1) Up to isomorphism, there are 321,931 non-degenerate involutive settheoretic solutions to the Yang-Baxter equation of size nine.

(2) Up to isomorphism, there are 4,895,272 non-degenerate involutive set-theoretic solutions to the Yang-Baxter equation of size ten.

Our methods can be easily adapted to construct racks of small size. Racks are particular types of solutions to the YBE that play a fundamental role in combinatorial knot theory, see Section 3.1. Using the 16,023 isomorphism classes of racks of size eight, we obtain the following result for non-involutive solutions of size eight. 
Theorem 1.4. There are 422,449,480 non-isomorphic non-degenerate noninvolutive set-theoretic solutions to the Yang-Baxter equation of size eight.

No previous constructions of non-involutive solutions were known.

Our methods could be used to construct solutions of other sizes. However, the number of non-involutive solutions of size nine is expected to be extremely big. With 16,023 racks of size eight we constructed 422,449,480 non-involutive solutions, so the number of non-involutive solutions of size nine is expected to be enormous, as there are 159,526 racks of size nine.

The paper is organized as follows. In Section 2 we compute the number of involutive solutions. This is done by using a constraint satisfaction program and the language of cycle sets. The algorithm is described at the beginning of the section. As an application we enumerate several types of solutions such as indecomposable, irretractable and multipermutation solutions. We also enumerate counterexamples to a well-known conjecture of Gateva-Ivanova [17]. Finally, in Section 3 we use a similar algorithm and the same computational techniques to enumerate racks, non-involutive solutions and, in particular, non-involutive biquandles.

\section{INVOLUTIVE SOLUTIONS}

A solution $(X, r)$ is said to be involutive if $r^{2}=\mathrm{id}$. An involutive solution $(X, r)$ is said to be irretractable if $\tau_{x} \neq \tau_{y}$ for all $x \neq y$. Note that this is equivalent to $\sigma_{x} \neq \sigma_{y}$ for all $x \neq y$, as $T \sigma_{x} T^{-1}=\tau_{x}^{-1}$ holds for all $x \in X$, where $T: X \rightarrow X, T(x)=\tau_{x}^{-1}(x)$, see [13, Proposition 2.2]. An involutive solution $(X, r)$ is said to be square-free if $T(x)=x$ for all $x \in X$, or equivalently if $r(x, x)=(x, x)$ for all $x \in X$.

If $(X, r)$ is an involutive solution, we consider over $X$ the equivalence relation given by

$$
x \sim y \Longleftrightarrow \tau_{x}=\tau_{y} .
$$

This equivalence relation induces an involutive solution over the set of equivalence classes $X / \sim$, known as the retraction $\operatorname{Ret}(X, r)$ of $(X, r)$. An involutive solution $(X, r)$ is a multipermutation solution if there exists $n$ such that $\left|\operatorname{Ret}^{n}(X, r)\right|=1$, where $\operatorname{Ret}^{n+1}(X, r)=\operatorname{Ret}\left(\operatorname{Ret}^{n}(X, r)\right)$. Multipermutation solutions generalize those in Example 1.1.

The permutation group of an involutive solution $(X, r)$ is defined as the subgroup $\mathcal{G}(X, r)$ of $\operatorname{Sym}_{X}$ generated by the set $\left\{\tau_{x}: x \in X\right\}$. An involutive solution $(X, r)$ is said to be indecomposable if the group $\mathcal{G}(X, r)$ acts transitively on $X$ and decomposable otherwise.

To construct all isomorphism classes of non-degenerate involutive solutions, we will use the language of cycle sets, introduced by Rump in [33]. A cycle set is a pair $(X, \cdot)$, where $X$ is a set and $X \times X \rightarrow X,(x, y) \mapsto x \cdot y$, is a binary operation such that the following conditions are satisfied:

(1) Each map $\varphi_{x}: X \rightarrow X, y \mapsto x \cdot y$, is bijective, and

(2) $(x \cdot y) \cdot(x \cdot z)=(y \cdot x) \cdot(y \cdot z)$ for all $x, y, z \in X$.

A cycle set $(X, \cdot)$ is said to be non-degenerate if the map $X \rightarrow X, x \mapsto x \cdot x$, is bijective. Rump proved that non-degenerate involutive solutions are in bijective correspondence with non-degenerate cycle sets, i.e.

\{non-degenerate involutive solutions $\} \longleftrightarrow$ non-degenerate cycle sets\}. 
The correspondence is given by the following formulas: If $(X, r)$ is a solution, then $(X, \cdot)$, where $x \cdot y=\tau_{x}^{-1}(y)$, is a non-degenerate cycle set. Conversely, if $(X, \cdot)$ is a cycle set, then $(X, r)$, where

$$
r(x, y)=((y * x) \cdot y, y * x)
$$

is a non-degenerate involutive solution, where $y * x=z$ if and only if $y \cdot z=x$.

Example 2.1. Let $X=\{1,2,3\}$ and $r(x, y)=\left(\sigma_{x}(y), \tau_{y}(x)\right)$, where

$$
\sigma_{1}=\mathrm{id}, \quad \sigma_{2}=\sigma_{3}=(23), \quad \tau_{1}=\tau_{2}=\tau_{3}=(23) .
$$

This solution is involutive, decomposable and multipermutation. The map $T: X \rightarrow X$ is the permutation (23). The cycle set associated to $(X, r)$ is given by the permutations

$$
\varphi_{1}=\mathrm{id}, \quad \varphi_{2}=(23), \quad \varphi_{3}=(23),
$$

Example 2.2. Let $X=\{1,2,3,4\}$ and $r(x, y)=\left(\sigma_{x}(y), \tau_{y}(x)\right)$, where

$$
\begin{aligned}
& \sigma_{1}=(34), \quad \sigma_{2}=(1324), \quad \sigma_{3}=(1423), \quad \sigma_{4}=(12), \\
& \tau_{1}=(24), \quad \tau_{2}=(1432), \quad \tau_{3}=(1234), \quad \tau_{4}=(13) .
\end{aligned}
$$

This involutive solution is irretractable, so it is not multipermutation. The permutation group of $(X, r)$ is $\mathcal{G}(X, r)=\langle(34),(1324),(1423),(12)\rangle \simeq \mathbb{D}_{4}$, the dihedral group of order eight. This group acts transitively on $X$, so $(X, r)$ is indecomposable. Note that $T: X \rightarrow X$ is the permutation (23). The cycle set associated to $(X, r)$ is given by the permutations

$$
\varphi_{1}=(24), \quad \varphi_{2}=(1234), \quad \varphi_{3}=(1432), \quad \varphi_{4}=(13) .
$$

The solutions $(X, r)$ and $(Y, s)$ are isomorphic if and only if their associated cycle sets are isomorphic, which means that there is a bijective map $f: X \rightarrow Y$ such that $f\left(x_{1} \cdot x_{2}\right)=f\left(x_{1}\right) \cdot f\left(x_{2}\right)$ for all $x_{1}, x_{2} \in X$. Note that one can write this formula as

$$
f \varphi_{x} f^{-1}=\varphi_{f(x)}
$$

for all $x \in X$.

One can translate the definitions given at the beginning of the section in the language of cycle sets. For example, the permutation group of a cycle set $(X, \cdot)$ is then defined as the group generated by the set $\left\{\varphi_{x}: x \in X\right\}$, and a cycle set is said to be indecomposable (resp. decomposable) if its permutation group acts transitively (resp. intransitively) on $X$.

For a cycle set $(X, \cdot)$ let $T: X \rightarrow X$ be the map given by $T(x)=x \cdot x$. By definition, the cycle set is non-degenerate if and only if the map $T$ is bijective. In [13, Proposition 2.2], Etingof, Schedler and Soloviev proved that $T$ is always bijective whenever the solution is finite, thus finite cycle sets are regular. This was proved independently by Rump in [33].

A cycle set $(X, \cdot)$, where $X=\{1,2, \ldots, n\}$, is encoded in a table

$$
M=\left(M_{i, j}\right)_{1 \leqslant i, j \leqslant n}, \quad M_{i, j}=\varphi_{i}(j)=i \cdot j .
$$

This means that the rows of $M$ are the permutations $\varphi_{1}, \ldots, \varphi_{n}$ defining the cycle set structure on $X$. The principal diagonal of $M$ is precisely the bijective map $T: X \rightarrow X, x \mapsto x \cdot x$. 
Example 2.3. The cycle set corresponding to the solution of Example 2.1 can be described by the matrix

$$
M=\left(\begin{array}{lll}
1 & 2 & 3 \\
1 & 3 & 2 \\
1 & 3 & 2
\end{array}\right) .
$$

Example 2.4. The cycle set corresponding to the solution of Example 2.2 can be described by the matrix

$$
M=\left(\begin{array}{llll}
1 & 4 & 3 & 2 \\
2 & 3 & 4 & 1 \\
4 & 1 & 2 & 3 \\
3 & 2 & 1 & 4
\end{array}\right)
$$

To construct all involutive solutions we need to find all possible matrices $M \in \mathbb{Z}^{n \times n}$ with coefficients in $\{1,2, \ldots, n\}$ such that

(1) for each $i$ the elements $M_{i, j}$ are all different,

(2) the elements of the principal diagonal of $M$ are all different, and

(3) $M_{M_{i, j}, M_{i, k}}=M_{M_{j, i}, M_{j, k}}$ holds for all $i, j, k \in\{1, \ldots, n\}$.

Since the map $T$ is bijective, the diagonal $\left(M_{i, i}\right)_{1 \leqslant i \leqslant n}$ has $n$ different elements. This fact is used to reduce our search space. The general idea goes back to Plemmons [32], but in our particular case is based on the following lemma:

Lemma 2.5. Let $n \in \mathbb{N}$ and $(X, \cdot)$ be a cycle set of size $n$. Let $T: X \rightarrow X$, $T(x)=x \cdot x$ and $T_{1} \in \operatorname{Sym}_{n}$. If $T_{1}$ and $T$ are conjugate, then there exists $a$ cycle set structure $\bullet$ on $X$ such that $(X, \bullet) \simeq(X, \cdot)$ and $T_{1}(x)=x \bullet x$ for all $x \in X$.

Proof. Let $\gamma \in \operatorname{Sym}_{n}$ be such that $T_{1}=\gamma^{-1} T \gamma$. A direct calculation shows that the operation $y \bullet z=\gamma^{-1}(\gamma(y) \cdot \gamma(z))$ turns $X$ into a cycle set isomorphic to $(X, \cdot)$ and such that

$$
y \bullet y=\gamma^{-1}(\gamma(y) \cdot \gamma(y))=\gamma^{-1}(T(\gamma(y)))=\left(\gamma^{-1} T \gamma\right)(y)
$$

holds for all $y \in X$.

Lemma 2.5 implies that there are only a small number of diagonals to consider, each diagonal being a representative of a conjugacy class in the symmetric group $\mathrm{Sym}_{n}$. Thus the original problem is divided into $p(n)$ problems, where $p(n)$ is the number of partitions of $n$. In the particular case of solutions of size nine, this means that there are $p(9)=30$ independent cases to consider. For size ten, there are $p(10)=42$ independent cases to consider.

To construct non-isomorphic solutions we shall need the following notation: If $g \in \operatorname{Sym}_{n}$ and $M$ is a matrix, we denote by $M^{g}$ the matrix given by

$$
\left(M^{g}\right)_{i, j}=g^{-1}\left(M_{g(i), g(j)}\right)
$$

for all $i, j \in\{1, \ldots, n\}$. To avoid expensive isomorphism checking, we are interested in those matrices $M$ such that

$$
M \leqslant_{\operatorname{lex}} M^{g}
$$


for all $g$ in the centralizer $C_{\operatorname{Sym}_{n}}(T)$ of the permutation $T$ in $\operatorname{Sym}_{n}$, where lex stands for the lexicographic ordering given by $A \leqslant \operatorname{lex} B$ if and only if

$$
\begin{aligned}
& \left(A_{1,1}, A_{1,2}, \ldots, A_{1, n}, A_{2,1}, A_{2,2}, \ldots, A_{n, n}\right) \\
& \quad \leqslant\left(B_{1,1}, B_{1,2}, \ldots, B_{1, n}, B_{2,1}, B_{2,2}, \ldots, B_{n, n}\right)
\end{aligned}
$$

with lexicographical order. This symmetry breaking is in general very hard to implement, as in this case the number of constraints will be extremely large. This happens for example when $T=$ id or $T$ is a transposition. To deal with this problem, we consider the constraint (2.1) only for those permutations that belong to a certain subset $S$ of $\mathrm{Sym}_{n}$. This is called partial symmetry breaking, see Remark 2.9 below for details. It should be noted that the use of proper subsets of the centralizer of $T$ produce some superfluous solutions and hence some repetitions should be removed by other computational methods.

We briefly describe our method to remove repetitions. Constraint satisfaction methods produce a list of solutions. Among the solutions in this list, only those minimal in their orbits are needed. A GAP script parses the list and, for each solution, checks whether or not the solution is minimal with respect to the lexicographic ordering inside its orbit. This stage of the process is mostly related with permutation groups and, in general, GAP deals with them in a friendly and very efficient way.

The enumeration of involutive solutions of size $\leqslant 8$ first appeared in [13]. Table 2.1 shows some numbers corresponding to solutions of size $\leqslant 10$. New results are presented in shaded cells. It should be noted that our numbers differ sightly from those of [13, Table 1], as our table contains two solutions of size eight that are not present in previous calculations.

Our approach with constraint programming needs about ten minutes to construct all those solutions of size $\leqslant 8$ up to isomorphism. The calculations for solutions of size nine took less than four hours and for size ten it took several days, see Tables 2.2, 2.3 and 2.4 for some runtimes. They were both performed in an Intel(R) Core(TM) i7-8700 CPU @ 3.20GHz, with 32GB RAM. The database of involutive solutions of size $\leqslant 9$ needs about $90 \mathrm{MB}$. Almost $2 \mathrm{~GB}$ are needed to store all involutive solutions of size 10 .

\begin{tabular}{|r|ccccccccc|}
\hline$n$ & 2 & 3 & 4 & 5 & 6 & 7 & 8 & 9 & 10 \\
\hline solutions & 2 & 5 & 23 & 88 & 595 & 3,456 & 34,530 & 321,931 & $4,895,272$ \\
square-free & 1 & 2 & 5 & 17 & 68 & 336 & 2,041 & 15,534 & 150,957 \\
ind. & 1 & 1 & 5 & 1 & 10 & 1 & 100 & 16 & 36 \\
m.p. & 2 & 5 & 21 & 84 & 554 & 3,295 & 32,155 & 305,916 & $4,606,440$ \\
irretractable & 0 & 0 & 2 & 4 & 9 & 13 & 191 & 685 & 3,590 \\
\hline
\end{tabular}

TABLE 2.1. Involutive solutions of size $\leqslant 10$.

For size $\leqslant 7$ our calculations coincide with those in [13], but differ by two for $n=8$ when the map $T$ an 8 -cycle (see Examples 2.6 and 2.7 below). We contacted the authors of [13] regarding the aforementioned discrepancy and they found the missing solutions after a re-run of their own code. 
Example 2.6. Let $X=\{1,2, \ldots, 8\}$ and $r(x, y)=\left(\sigma_{x}(y), \tau_{y}(x)\right)$, where

$$
\begin{array}{ll}
\sigma_{1}=\sigma_{5}=(16345278), & \sigma_{2}=\sigma_{6}=(12745638), \\
\sigma_{3}=\sigma_{7}=(12385674), & \sigma_{4}=\sigma_{8}=(16785234), \\
\tau_{1}=\tau_{5}=(18365472), & \tau_{2}=\tau_{6}=(14765832), \\
\tau_{3}=\tau_{7}=(14325876), & \tau_{4}=\tau_{8}=(18725436) .
\end{array}
$$

Then $(X, r)$ is an indecomposable and multipermutation involutive solution.

Example 2.7. Let $X=\{1,2, \ldots, 8\}$ and $r(x, y)=\left(\sigma_{x}(y), \tau_{y}(x)\right)$, where

$$
\begin{aligned}
& \sigma_{1}=\sigma_{5}=(1278)(3456), \quad \sigma_{2}=\sigma_{6}=(1238)(4567), \\
& \sigma_{3}=\sigma_{7}=(1234)(5678), \quad \sigma_{4}=\sigma_{8}=(1678)(2345), \\
& \tau_{1}=\tau_{5}=(1832)(4765), \quad \tau_{2}=\tau_{6}=(1432)(5876), \\
& \tau_{3}=\tau_{7}=(1876)(2543), \quad \tau_{4}=\tau_{8}=(1872)(3654) \text {. }
\end{aligned}
$$

Then $(X, r)$ is an indecomposable and multipermutation involutive solution.

Remark 2.8. The involutive solutions of Examples 2.6 and 2.7 are multipermutation and indecomposable solutions. This means that there are 34,530 solutions of size eight, 100 of them are indecomposable and 39 are multipermutation and indecomposable.

Remark 2.9. As mentioned before, for some diagonals $T$ the centralizer turns out to be too big for our computational resources. A sample $S$ of elements of $C_{\mathrm{Sym}_{n}}(T)$ is to be chosen to make the constraint computations feasible. To construct solutions of size $n \in\{9,10\}$, taking $S$ as the full centralizer $C_{\mathrm{Sym}_{n}}(T)$ of the permutation $T$ in $\operatorname{Sym}_{n}$ works well for small centralizers. For big centralizers, as it is the case when $T=$ id or a transposition, the standard heuristic local search suggests to look at the subset of $C_{\mathrm{Sym}_{n}}(T)$ consisting of permutations moving a small number of points of $\{1,2, \ldots, n\}$ (at most three usually suffices), as most violations of the minimality condition involve few entries of the matrix. We also include a small generating set of $C_{\mathrm{Sym}_{n}}(T)$, since we do not want to lose information by inadvertently ignoring permutations that change certain labels. These particular choices of sets $S$ work well in our setting and allow us to construct solutions in a reasonable time. The partial symmetry breaking technique described in this paragraph and some of its variations were studied by McDonald and Smith in [28] and the automation of these techniques were studied by Jefferson and Petrie in [24]. 
Ö. AKGÜN, M. MEREB, L. VENDRAMIN

\begin{tabular}{|c|c|c|c|}
\hline$n$ & $T$ & Solutions & CPU time \\
\hline 9 & $(123456789)$ & 9 & 3 minutes \\
& $(12345678)$ & 104 & 6 minutes \\
& $(1234567)$ & 35 & 2 minutes \\
& $(123456)$ & 1,176 & 2 minutes \\
\hline 10 & $(123456789 \mathrm{a})$ & 20 & 10 hours \\
& $(123456789)$ & 81 & 11 hours \\
& $(12345678)$ & 720 & 9 hours \\
& $(1234567)$ & 238 & 2 hours \\
& $(123456)$ & 9,103 & 2 hours \\
\hline
\end{tabular}

TABLE 2.2. Some runtimes for constructing involutive solutions of size $n \in\{9,10\}$ with $S=C_{\text {Sym }_{n}}(T)$. In these cases there is no need to check if some solutions are isomorphic.

\begin{tabular}{|c|c|c|c|}
\hline$n$ & $T$ & Solutions & CPU time \\
\hline 9 & $(12345)$ & 780 & 2 minutes \\
& $(1234)$ & 11,320 & 3 minutes \\
& $(123)$ & 13,061 & 4 minutes \\
& $(12)(34)(56)(78)$ & 24,345 & 6 minutes \\
& $(12)(34)(56)$ & 52,866 & 4 minutes \\
& $(12)(34)$ & 61,438 & 8 minutes \\
& $(12)$ & 41,732 & 50 minutes \\
\hline
\end{tabular}

TABLE 2.3. Some runtimes for constructing involutive solutions of size nine with $S$ being a generating set of $C_{\mathrm{Sym}_{n}}(T)$.

\begin{tabular}{|c|c|c|c|}
\hline$n$ & $T$ & Solutions & CPU time \\
\hline 9 & $(12345)$ & 780 & 1 minute \\
& $(1234)$ & 11,320 & 1 minute \\
& $(123)$ & 13,061 & 2 minutes \\
& $(12)(34)(56)(78)$ & 24,345 & 17 minutes \\
& $(12)(34)(56)$ & 52,866 & 9 minutes \\
& $(12)(34)$ & 61,438 & 7 minutes \\
& $(12)$ & 41,732 & 11 minutes \\
& id & 15,534 & 1 hour 20 minutes \\
\hline 10 & $(123)$ & 143,267 & 2 days \\
& $(12)(34)(56)(78)(9 \mathrm{a})$ & 178,782 & 2 days 7 hours \\
& $(12)(34)(56)(78)$ & 560,592 & 2 days \\
& $(12)(34)(56)$ & 855,536 & 10 hours \\
& $(12)(34)$ & 807,084 & 8 hours \\
& $(12)$ & 474,153 & 17 hours \\
& id & 150,957 & 6 days \\
\hline
\end{tabular}

TABLE 2.4. Some runtimes for constructing involutive solutions of size $n \in\{9,10\}$. In these cases $S$ is the set of permutations of $C_{\mathrm{Sym}_{n}}(T)$ that move $\leqslant 3$ points. 
In [17] Gateva-Ivanova conjectured that all finite square-free solutions are retractable. Despite the fact that the conjecture holds in several cases (see [1, $8,20,29]$ ) a counterexample was found in [39]. From a given counterexample one then constructs other counterexamples by different methods, see [3, 7]. It turns out that constructing essentially new counterexamples to the conjecture seems to be quite challenging.

For $n \in \mathbb{N}$ let $g(n)$ be the number of isomorphism classes of counterexamples to Gateva-Ivanova conjecture. Computer calculations show that $g(n)=0$ if $n \leqslant 7$. Other values of $g(n)$ are shown in Table 2.5.

\begin{tabular}{|c|cccc|}
\hline$n$ & 8 & 9 & 10 & 11 \\
\hline$g(n)$ & 1 & 5 & 12 & 23 \\
\hline
\end{tabular}

TABLE 2.5. Some values of $g(n)$.

The determination of the exact value of $g(9)$ took about 7 minutes, $g(10)$ took 3 hours and $g(11)$ took four days. The calculations were performed in an Intel(R) Xeon(R) CPU E5-2670, 2.60GHz, with 32GB RAM.

\section{Non-InVOLUTiVE SOLUTiOns}

The method presented in Section 2 is now used to compute non-involutive solutions. This time, we translate the problem into the language of skew cycle sets. First we need basic definitions of the theory of racks.

3.1. Racks. A rack is a pair $(X, \triangleright)$, where $X$ is a set and $X \times X \rightarrow X$, $(x, y) \mapsto x \triangleright y$, is a binary operation on $X$ such that the following conditions are satisfied:

(1) Each map $X \rightarrow X, y \mapsto x \triangleright y$ is bijective, and

(2) $x \triangleright(y \triangleright z)=(x \triangleright y) \triangleright(x \triangleright z)$ for all $x, y, z \in X$.

We can use the ideas presented in the previous section to construct finite racks up to isomorphisms. However, algorithms to construct and enumerate finite racks of small size are already known, see for example in $[2,6,23,40]$.

As we need racks to construct arbitrary solutions to the YBE, it is convenient to recall that the construction problem for racks can be formulated as follows: We need to find all matrices $R \in \mathbb{Z}^{n \times n}$ with coefficients in $\{1,2, \ldots, n\}$ such that

(1) for each $i$ the elements $R_{i, j}$ are all different,

(2) the elements of the principal diagonal of $R$ are all different, and

(3) $R_{i, R_{j, k}}=R_{R_{i, j}, R_{i, k}}$ holds for all $i, j, k \in\{1, \ldots, n\}$.

To construct racks we can use the trick of considering only representatives of conjugacy classes of the diagonal and then keep only those matrices which are minimal in their orbits, with respect to the lexicographical order.

For $n \in \mathbb{N}$, let $r(n)$ be the number of isomorphism classes of racks of size $n$. Some values of $r(n)$ appear in Table 3.1. These values of $r(n)$ were computed by our method based on constraint programming. A better approach to the enumeration of racks of small size appears in [40]. 


\begin{tabular}{|c|cccccccc|}
\hline$n$ & 2 & 3 & 4 & 5 & 6 & 7 & 8 & 9 \\
\hline$r(n)$ & 2 & 6 & 19 & 74 & 353 & 2,080 & 160,23 & 159,526 \\
\hline
\end{tabular}

TABLE 3.1. Enumeration of racks.

3.2. Non-involutive solutions. The theory of cycle sets can be generalized to deal with non-involutive solutions to the YBE, see for example [35]. A skew cycle set is a triple $(X, \cdot, \triangleright)$, where $(X, \triangleright)$ is a rack and $X \times X \rightarrow X$, $(x, y) \mapsto x \cdot y$, is a binary operation such that

(1) The maps $\varphi_{x}: X \rightarrow X, y \mapsto x \cdot y$, are bijective,

(2) $(x \cdot(x \triangleright y)) \cdot(x \cdot z)=(y \cdot x) \cdot(y \cdot z)$ for all $x, y, z \in X$, and

(3) $x \cdot(y \triangleright z)=(x \cdot y) \triangleright(x \cdot z)$ for all $x, y, z \in X$.

As it happens in the involutive case, finite solutions to the YBE are in bijective correspondence with skew cycle sets, i.e.

\{non-degenerate solutions $\} \longleftrightarrow$ nnon-degenerate skew cycle sets\}

The correspondence is given as follows. If $(X, r)$ is a solution, then the skew cycle set on $X$ is given by

$$
x \cdot y=\tau_{x}^{-1}(y), \quad x \triangleright y=\tau_{x} \sigma_{\tau_{y}^{-1}(x)}(y) .
$$

Conversely, if $X$ is a skew cycle set, then

$$
r(x, y)=((y * x) \cdot((y * x) \triangleright y), y * x)
$$

is a solution, where $y * x=z$ if and only if $y \cdot z=x$. We refer to [26] for more information on the interaction between solutions and their associated racks.

Remark 3.1. Under the bijective correspondence (3.1), involutive solutions correspond to the cycle sets defined in Section 2.

We now translate the problem of constructing all finite solutions into a problem suitable for constraint programming. Given a matrix $R$ corresponding to a rack of size $n$, we want to find all possible matrices $M \in \mathbb{Z}^{n \times n}$ with coefficients in $\{1,2, \ldots, n\}$ such that

(1) for each $i$ the elements $M_{i, j}$ are all different,

(2) the elements of the principal diagonal of $M$ are all different,

(3) $M_{M_{i, R_{i, j}}, M_{i, k}}=M_{M_{j, i}, M_{k, l}}$ holds for all $i, j, k \in\{1, \ldots, n\}$, and

(4) $M_{i, R_{j, k}}=R_{M_{i, j}, M_{i, k}}$ for all $i, j, k \in\{1, \ldots, n\}$.

We can exclude the trivial rack from our algorithm, as involutive solutions were computed in Section 2. It only remains to deal with the isomorphism problem. Thus we are interested in those matrices $M$ such that

$$
M \leqslant_{\operatorname{lex}} M^{g}
$$

for all $g$ in the stabilizer of the rack $R$, where lex stands for the lexicographic ordering on matrices described in Section 2. This symmetry breaking is in general easy to implement, as stabilizers of racks tend to be small.

For $n \in \mathbb{N}$ let $s(n)$ be the number of isomorphism classes of non-involutive solutions of size $n$. We summarize our calculations in Table 3.2. 


\begin{tabular}{|c|ccccccc|}
\hline$n$ & 2 & 3 & 4 & 5 & 6 & 7 & 8 \\
\hline$s(n)$ & 2 & 21 & 230 & 3,519 & 100,071 & $4,602,720$ & $422,449,480$ \\
\hline
\end{tabular}

TABLE 3.2. Enumeration of non-involutive solutions.

The calculations for $s(n)$ for all $n \leqslant 6$ took about 10 minutes, $s(7)$ needed 2 hours and 17 minutes and $s(8)$ took about one day. The database of noninvolutive solutions needs about $750 \mathrm{MB}$ for solutions of size $\leqslant 7$ and around 100GB for solutions of size eight.

3.3. Non-involutive biquandles. Recall that a biquandle is a solution such that its associated rack is a quandle, that means that

$$
x \triangleright x=\tau_{x} \sigma_{\tau_{x}^{-1}(x)}(x)=x
$$

for all $x \in X$. In particular, involutive solutions are biquandles. Enumeration of biquandles of small size appear in $[4,14,30]$.

For $n \in \mathbb{N}$ let $b(n)$ be the number of isomorphism classes of non-involutive biquandles of size $n$. The enumeration of non-involutive biquandles appear in Table 3.3.

\begin{tabular}{|c|cccccc|}
\hline$n$ & 3 & 4 & 5 & 6 & 7 & 8 \\
\hline$b(n)$ & 10 & 75 & 974 & 18,548 & 621,414 & $37,836,551$ \\
\hline
\end{tabular}

TABLE 3.3. Enumeration of non-involutive biquandles.

Acknowledgments. We thank A. Bartholomew, M. Farinati, P. Etingof and T. Schedler for useful conversations. The second author is partially supported by PICT 2018-3511 and is also a Junior Associate of the ICTP. The third author acknowledges support of NYU-ECNU Institute of Mathematical Sciences at NYU-Shanghai and he is supported in part by PICT 2016-2481 and UBACyT 20020170100256BA.

\section{REFERENCES}

[1] E. Acri, R. Lutowski, and L. Vendramin. Retractability of solutions to the YangBaxter equation and $p$-nilpotency of skew braces. Internat. J. Algebra Comput., 30(1):91-115, 2020.

[2] M. Ashford and O. Riordan. Counting racks of order n. Electron. J. Combin., 24(2):Paper No. 2.32, 20, 2017.

[3] D. Bachiller, F. Cedó, E. Jespers, and J. Okniński. A family of irretractable squarefree solutions of the Yang-Baxter equation. Forum Math., 29(6):1291-1306, 2017.

[4] A. Bartholomew and R. Fenn. Biquandles of small size and some invariants of virtual and welded knots. J. Knot Theory Ramifications, 20(7):943-954, 2011.

[5] R. J. Baxter. Partition function of the eight-vertex lattice model. Ann. Physics, 70:193-228, 1972.

[6] S. R. Blackburn. Enumerating finite racks, quandles and kei. Electron. J. Combin., 20(3):Paper 43, 9, 2013.

[7] M. Castelli, F. Catino, and G. Pinto. About a question of Gateva-Ivanova and Cameron on square-free set-theoretic solutions of the Yang-Baxter equation. Comm. Algebra, 48(6):2369-2381, 2020.

[8] F. Cedó, E. Jespers, and J. Okniński. Retractability of set theoretic solutions of the Yang-Baxter equation. Adv. Math., 224(6):2472-2484, 2010.

[9] L. N. Childs. Bi-skew braces and Hopf Galois structures. New York J. Math., 25:574$588,2019$. 
[10] K. De Commer. Actions of skew braces and set-theoretic solutions of the reflection equation. Proc. Edinb. Math. Soc. (2), 62(4):1089-1113, 2019.

[11] A. Distler, C. A. Jefferson, T. Kelsey, and L. Kotthoff. The Semigroups of Order 10. In 18th International Conference on Principles and Practice of Constraint Programming, pages 883-899, Oct. 2012.

[12] V. G. Drinfel'd. On some unsolved problems in quantum group theory. In Quantum groups (Leningrad, 1990), volume 1510 of Lecture Notes in Math., pages 1-8. Springer, Berlin, 1992.

[13] P. Etingof, T. Schedler, and A. Soloviev. Set-theoretical solutions to the quantum Yang-Baxter equation. Duke Math. J., 100(2):169-209, 1999.

[14] R. A. Fenn and A. Bartholomew. Erratum: Biquandles of small size and some invariants of virtual and welded knots [ MR2819176]. J. Knot Theory Ramifications, 26(8):1792002, 11, 2017.

[15] A. M. Frisch, W. Harvey, C. Jefferson, B. Martínez-Hernández, and I. Miguel. Essence: A constraint language for specifying combinatorial problems. Constraints, 13(3):268-306, Sept. 2008.

[16] The GAP Group. GAP - Groups, Algorithms, and Programming, Version 4.11.0, 2020.

[17] T. Gateva-Ivanova. A combinatorial approach to the set-theoretic solutions of the Yang-Baxter equation. J. Math. Phys., 45(10):3828-3858, 2004.

[18] T. Gateva-Ivanova. Quadratic algebras, Yang-Baxter equation, and Artin-Schelter regularity. Adv. Math., 230(4-6):2152-2175, 2012.

[19] T. Gateva-Ivanova. Set-theoretic solutions of the Yang-Baxter equation, braces and symmetric groups. Adv. Math., 338:649-701, 2018.

[20] T. Gateva-Ivanova and P. Cameron. Multipermutation solutions of the Yang-Baxter equation. Comm. Math. Phys., 309(3):583-621, 2012.

[21] T. Gateva-Ivanova and M. Van den Bergh. Semigroups of I-type. J. Algebra, 206(1):97-112, 1998.

[22] I. P. Gent, C. Jefferson, and I. Miguel. Minion: A fast, scalable, constraint solver. In Proceedings of the 2006 Conference on ECAI 2006: 17th European Conference on Artificial Intelligence August 29 - September 1, 2006, Riva Del Garda, Italy, page 98-102, NLD, 2006. IOS Press.

[23] J. Hoste and P. D. Shanahan. An enumeration process for racks. Math. Comp., 88(317):1427-1448, 2019.

[24] C. Jefferson and K. E. Petrie. Automatic generation of constraints for partial symmetry breaking. In J. Lee, editor, Principles and Practice of Constraint Programming CP 2011, pages 729-743, Berlin, Heidelberg, 2011. Springer Berlin Heidelberg.

[25] V. Lebed and L. Vendramin. Homology of left non-degenerate set-theoretic solutions to the Yang-Baxter equation. Adv. Math., 304:1219-1261, 2017.

[26] V. Lebed and L. Vendramin. On structure groups of set-theoretic solutions to the Yang-Baxter equation. Proc. Edinb. Math. Soc. (2), 62(3):683-717, 2019.

[27] J.-H. Lu, M. Yan, and Y.-C. Zhu. On the set-theoretical Yang-Baxter equation. Duke Math. J., 104(1):1-18, 2000.

[28] I. McDonald and B. Smith. Partial symmetry breaking. In International Conference on Principles and Practice of Constraint Programming, pages 431-445. Springer, 2002.

[29] H. Meng, A. Ballester-Bolinches, and R. Esteban-Romero. Left braces and the quantum Yang-Baxter equation. Proc. Edinb. Math. Soc. (2), 62(2):595-608, 2019.

[30] S. Nelson and J. Vo. Matrices and finite biquandles. Homology Homotopy Appl., $8(2): 51-73,2006$

[31] P. Nightingale, Ö. Akgün, I. P. Gent, C. Jefferson, I. Miguel, and P. Spracklen. Automatically improving constraint models in Savile Row. Artificial Intelligence, 251:3561, 2017.

[32] R. Plemmons. Construction and analysis of non-equivalent finite semigroups. In Computational Problems in Abstract Algebra (Proc. Conf., Oxford, 1967), pages 223-228. Pergamon, Oxford, 1970. 
[33] W. Rump. A decomposition theorem for square-free unitary solutions of the quantum Yang-Baxter equation. Adv. Math., 193(1):40-55, 2005.

[34] W. Rump. The brace of a classical group. Note Mat., 34(1):115-144, 2014.

[35] W. Rump. A covering theory for non-involutive set-theoretic solutions to the YangBaxter equation. J. Algebra, 520:136-170, 2019.

[36] A. Smoktunowicz and L. Vendramin. On skew braces (with an appendix by N. Byott and L. Vendramin). J. Comb. Algebra, 2(1):47-86, 2018.

[37] A. Soloviev. Non-unitary set-theoretical solutions to the quantum Yang-Baxter equation. Math. Res. Lett., 7(5-6):577-596, 2000.

[38] M. Takeuchi. Survey on matched pairs of groups - an elementary approach to the ESS-LYZ theory. In Noncommutative geometry and quantum groups (Warsaw, 2001), volume 61 of Banach Center Publ., pages 305-331. Polish Acad. Sci., Warsaw, 2003.

[39] L. Vendramin. Extensions of set-theoretic solutions of the Yang-Baxter equation and a conjecture of Gateva-Ivanova. J. Pure Appl. Algebra, 220(5):2064-2076, 2016.

[40] P. Vojtěchovský and S. Y. Yang. Enumeration of racks and quandles up to isomorphism. Math. Comp., 88(319):2523-2540, 2019.

[41] C. N. Yang. Some exact results for the many-body problem in one dimension with repulsive delta-function interaction. Phys. Rev. Lett., 19:1312-1315, 1967.

(M. Mereb and L. Vendramin) IMAS-CONICET and Depto. de Matemática, FCEn, Universidad de Buenos Aires, Pab. 1, Ciudad Universitaria, C1428EGA, Buenos Aires, Argentina

Email address: lvendramin@dm.uba.ar

Email address: mmereb@dm.uba.ar

(L. Vendramin) Department of Mathematics, Vrije Universiteit Brussel, Plein-】 LAAN 2, 1050 Brussel, BELgiUm

Email address: Leandro.Vendramin@vub.be

School of Computer Science, University of St Andrews, St Andrews, Fife KY16 9SX, UK.

Email address: ozgur.akgun@st-andrews.ac.uk 